\title{
HOMOGENIZACIÓN DE LA CULTURA ALIMENTARIA ENTRE NIÑOS Y ADOLESCENTES
}

\author{
Reig MF*, Rizo $\mathrm{MM}^{* *}$, Alarcón $\mathrm{N}^{*}$, Cortes $\mathrm{E}^{* * *}$ \\ * Diplomados en Nutrición Humana y Dietética \\ ** Profesora Enfermería. Universidad de Alicante \\ *** Profesor departamento pediatría. Universidad Miguel Hernández
}

\section{TOWARDS COMMON EATING HABITS IN CHILDREN AND TEENAGERS}

\section{ABSTRACT}

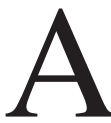
study was made about the eating habits of 104 children (12 and 18 years old), in different primary and secondary schools in the urban area of Alicante and the rural area of Murcia.

The study took place during the months of March, April and May, taking into consideration there was not exam periods. None of the children studied suffered from an illness which may had influenced their eating habits.

For the purpose of the study a weekly survey in which the children wrote their daily food intake was used. Only the surveys with full data were included in the study.

Low intake of fish, fruits and vegetables among the subjects was detected. It was also remarkable the low intake of carbohydrates, an increase in the intake of proteins and a decrease in the energy intake recommendable for their age. A change in the eating habits was common for both of the studied groups (rural and urban), becoming equal as an effect of cultural homogeneity, whose extends involves children and teenagers. Despite the fact that weekly surveys are not useful for evaluating the exact quantity of food intake, such as added fat, its use is recommended for detecting deviations more accurately rather than 24 hour surveys.

\section{RESUMEN}

e han estudiado los hábitos de alimentación de 104 niños de 12 años y adolescentes de 18 años, entre colegios e institutos de un entorno urbano de la provincia de Alicante, y un entorno rural de la provincia de Murcia.
El período de tiempo en que se realizó el estudio oscila entre marzo, abril y mayo. Ninguno de los niños manifestaba patología que influyera en los hábitos de alimentación, ni se encontraban en el período de exámenes.

Para este estudio se utilizó el carnet alimentario (Moreiras O.1990, Rizo M.1990) que es una encuesta abierta de siete días donde el niño y el adolescente anotan cada uno de los alimentos que ingieren de forma diaria, aclarando cualquier duda posible del día anterior por medio de los observadores. Se seleccionaron sólo los que tenía cumplimentados todos los parámetros de los carnet.

La totalidad de los datos de cada individuo se introdujeron en una base de datos del programa informático "SPSS" versión 11.5. Y por último, la utilización del programa "Alimentación y Salud" (Mataix.1995.Versión 0698.046) donde se ha valorado la ingesta de los alimentos y se ha obtenido una relación de ingesta de alimentos ricos en proteínas, grasas e hidratos de carbono.

Como resultados del estudio destacar el bajo consumo de pescado, frutas y verduras; dentro de los grupos de alimentos, se destaca el bajo consumo de los alimentos ricos en hidratos de carbono (patata, pan blanco, fruta...), el aumento de alimentos ricos en proteínas (Moreiras O.1989,Rizo M.2001) y la disminución de energía recomendable para su edad. Las modificaciones de los hábitos alimentarios fueron comunes a las dos poblaciones rural y urbana, igualándose éstos entre ambas poblaciones como efecto de la uniformidad cultural, efectos extensibles a los niños y adolescentes. Y por último, a pesar de que los carnet alimentarios de una semana no valoran las cantidades de los alimentos, como las ingesta de grasas añadidas, pueden servir para detectar desviaciones poblacionales con una mayor exactitud que los estudios de 24 horas (Hetzler AA.1993,Jhonson RK.1991). 


\section{INTRODUCCIÓN}

La mejora en la salud y en la esperanza de vida que se ha dado en el último siglo en los países más industrializados se inició antes de la aparición de muchos de los avances médicos con los que se cuenta actualmente, dato que indica que dicha mejora en la salud fue debida principalmente a una mejora en las condiciones de vida. En la última década se ha puesto de manifiesto el hecho de que en muchas enfermedades no infecciosas intervienen también factores ambientales y que estas enfermedades pueden ser potencialmente prevenidas mediante cambios en las condiciones y/o hábitos de vida. De todos los factores ambientales que pueden influir en el estado de salud, la dieta es quizá el más importante, y esto explica que en las últimas décadas se haya despertado una preocupación creciente por la nutrición y los hábitos alimentarios de la población (Tojo R 2001). La alimentación no es sólo una respuesta fisiológica sino más o menos forma parte de los hábitos tradicionales modulados: moda, religión, prestigio... (Castells M. 1995).

En España, disfrutamos de una esperanza de vida relativamente alta en comparación con otros países. Esto se interpreta de modo que puede ser debido, al menos en parte, a la existencia de un perfil dietético particularmente saludable (MartiHenneberg 1995). Sin embargo, la población española ha cambiado sus hábitos de conducta en las últimas décadas, adquiriendo hábitos alimentarios y nutricionales que han sustituido a ciertas características culturales propias (Castell M 1995, MartíHenneberg C 1987, Moreiras o 1990, Serra L 1993, Rizo MM 2001, Rodríguez F 1996, Sabate F 1984). Dichas modificaciones no tanto en cuanto a consumo energético total, sino en cuanto a porcentajes de proteína de origen animal (Barrabriga A.1998, Rizo M.1990, Rizo M.2001, Rizo M. 2002). Han controlado que se esta disminuyendo la ingesta de los hidratos de carbono de cadena larga, de proteínas vegetales, de pescado y de fibra alimentaria. Así, en las décadas de los 60 a los 90, el consumo de carne de cerdo en España se ha multiplicado por tres, mientras que el aceite de oliva se consume la mitad (Barrabriga A 1998, Rizo MM 1988, Rizo MM 2002).

Estas variaciones han podido ser las causas de estos cambios en la alimentación, así según
Contreras (Contreras J.1993), pueden aplicarse las mismas que las descritas por Brewster y Jacobson (Brewster L 1978) para EEUU: nuevas tecnologías de conservación, aumento de poder adquisitivo, programas gubernamentales de subvención, trabajo de los dos miembros de la pareja, final del "baby boom" con un aumento de la edad media de la población, recomendaciones sanitarias, restaurantes de comida rápida y grandes campañas publicitarias. Sobre estas causas ahonda Gracia (Gracia M.1996) que indica que los cambios de los hábitos de alimentación pueden estar producidos por las variaciones en la transmisión oral madre a hija, mediante los medios de comunicación con sus mensajes que conforman el triángulo salud, estética y gastronomía.

Si además se tiene en cuenta que en España, cerca de la mitad de la población muere por enfermedades cardiovasculares, y que lesiones ateroscleróticas en adulto son el estado final de lesiones que se iniciaron en la infancia (Alonso de Armiño V. 1991), se hace necesaria una actuación sobre los niños y las familias, desde la escuela y centros de salud principalmente, que sea coherente y en una línea uniforme (Maldonado P 1996). En el presente estudio se persigue verificar las similitudes o diferencias entre los hábitos de alimentación de niños y adolescentes en una población urbana y rural; adquiriendo los máximos conocimientos sobre los hábitos actuales de alimentación en adolescentes con la implantación de la "comida rápida".

\section{MATERIAL Y MÉTODO Población de estudio:}

Se han estudiado los hábitos de alimentación de 104 niños y adolescentes, entre ellos niños y adolescentes de colegios e institutos de un entorno urbano de la provincia de Alicante (Villena), y un entorno rural de la provincia de Murcia (Llano de Brujas). En los niños de edades de 12 a 13 años se utilizó uno de los colegios del entorno urbano y el único colegio existente en el entorno rural; y en los adolescentes de 14 a 18 años se realizó en los institutos del entorno urbano y en el instituto del entorno rural, con la diferencia de que se escogió dos clases en este último para comparar en igualdad de condiciones por la selección del total de dos clases en otro entorno (tabla 1). 
El período de tiempo en que se realizó el estudio oscila entre marzo, abril y mayo. Ninguno de los niños manifestaba patología que influyera en los hábitos de alimentación y los adolescentes no se encontraban en el período de exámenes.

El estudio se efectuó en una población urbana de la provincia de Alicante de 32.282 habitantes y en una población rural de la Región de Murcia con 4.632 habitantes.

\section{Metodología empleada}

Se seleccionaron los centros con el permiso pertinente, impartiendo un taller donde se les explicó unas pequeñas nociones básicas de nutrición y cómo se rellenaba el carnet alimentario. Los conocimientos de nutrición fueron valorados mediante el método "Plenufar", con preguntas como "¿sabes lo que son las pastas, los cereales ...?".

Se valoraron de las nociones de nutrición sobre la conducta alimentaria de la anorexia y bulimia nerviosa tanto en niños y adolescentes. Para esta valoración se utilizó un test sobre la posible consideración como alumnos de riesgo sobre el tema en cuestión. Se diseñó un test con algunas preguntas que se dirigen a pacientes con anorexia o bulimia nerviosa por adaptarlo al público al que se refería (Alarcón N 2001).

Para este estudio se utilizó el carnet alimentario (Moreiras O.1990, Rizo M.1990) durante siete días abierta donde el niño y el adolescente anotan cada uno de los alimentos que ingieren de forma diaria, aclarando cualquier duda posible del día anterior por medio de los observadores. Los dos observadores han hecho el trabajo de campo juntos con los mismos criterios de evaluación.

Se seleccionaron sólo los que tenía cumplimentados todos los parámetros de los carnets alimentarios.

La totalidad de los datos de cada individuo se introdujeron en una base de datos del programa informático "SPSS" Versión 11.5, y se obtuvo el estudio estadístico. Las variables que se utilizaron a la hora de la comparación son la edad, el ambiente rural o urbano, peso, altura, IMC, número de comidas y los distintos alimentos como: lácteos, huevos, jamón serrano, jamón de york, carne, pescado, pan, legumbres, patata, arroz, pasta, dulces, bollería, chocolate, embutido, fruta, verdura, zumos, refrescos, aceites vegetales, mantequillas, cereales y frutos secos. También se aplicó este programa estadístico para tratar los resultados de la calidad de las distintas comidas (tabla 4) y para visualizar con quién y donde comen los individuos (tabla 5).

Los estadísticos utilizados han sido la media, la desviación estándar(SD) y la comparación entre los grupos mediante el test para muestras no paramétricas de Mann-Whitney, considerándo diferencias significativas para una $\mathrm{p}<0.05$.

Por último, los datos obtenidos por el tratamiento estadístico fueron introducidos en el programa "Alimentación y Salud" (Mataix. versión 0698.046), donde se ha valorado la ingesta de los alimentos y se ha obtenido una relación de ingesta de alimentos ricos en proteínas, grasas e hidratos de carbono.

\section{RESULTADOS}

Lo primero que se realizó una comparación por edades, y segundo una comparación por entornos. En la exposición de los resultados se consideró muy interesante resaltar la misma línea con que se contrastaron los datos a la hora de su valoración.

En los hábitos de alimentación de los niños de 12 años se observó la existencia de una alimentación inadecuada, donde se puede manejar la posi-

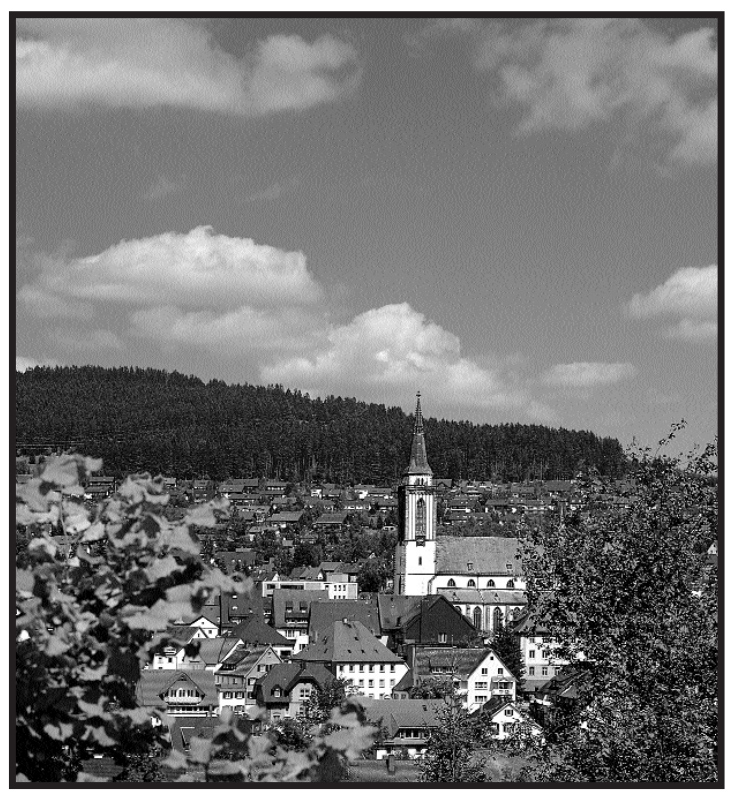


bilidad de que se buscara la comodidad y la rapidez de los padres en contraposición de unas buenas costumbres para los niños en crecimiento. Se observa que los desayunos rápidos con la ingesta de un solo lácteo o derivado e inclusive un zumo en ocasiones combinados con galletas o cereales; los almuerzos con bocadillos; las meriendas y las meriendas-cenas con bocadillos; las cenas con combinación de platos; y las colaciones con un lácteo sumado en ocasiones a galletas o cereales (tabla 4). Estas comidas se realizaron como norma general en casa con las siguientes compañías como preferencia: la familia al completo (padre, madre y hermanos), la madre, sólo, el padre y los hermanos (tabla 5). En las comidas fuera de casa las preferencias de compañías son: amigos, familiares (tíos/as) y abuelos, claro está, en lugares como el colegio, parques, casa de familiares y casa de los abuelos (tabla 5).

Las costumbres alimentarias de los niños estudiados en el entorno rural parecen ser más adecuadas por diversos motivos: 1) la relación de macronutrientes, solo se altera por una mayor ingesta de alimentos ricos en proteínas, un $20 \%$ cuando la normalidad en la ingesta es de 10-15\% (tabla 3), cuestión que es propia de la mejora económica de un país desarrollado (Medina FX 1996). 2) Las recomendaciones de hidratos de carbono en parte por el mayor consumo de patata y de pan, todo englobado en un marco de comidas en casa donde se justificaría el consumo de patata en comidas de olla y de desayunos recomendados por los padres, que les indicarían las mejores actuaciones; y 3) las comidas se han distribuido en 4 sesiones al día. Se aprecia también la sin justificación aparente el mayor consumo de refrescos. También se destaca la ingesta de colesterol en su alimentación es elevada. Y por último, la relación de peso y altura dará un IMC de P90, o sea, que estos niños superan la normalidad.

La alimentación de los niños en el entorno urbano se podría observar como valoración de mayor comodidad y rapidez en las comidas, ligado a un efecto de socialización más potenciado, con el mayor consumo de comida rápida tipo pizzas, hamburguesas y otras (3), que provoca un aumento en el consumo de grasas saturadas (tabla 3). Por último, hay que resaltar la mayor presencia de indivi- duos del sexo femenino, a pesar de que en el entorno rural también impera el mismo sexo (tabla 1).

Los adolescentes no plasman unas costumbres en la alimentación excesivamente deformadas como popularmente se comenta (tabla 2), aunque cabe la posibilidad de que estén afectados por la globalización y la publicidad (Castells M 1995) puesto que ambos grupos se aprecia una tendencia a la baja de los alimentos ricos en hidratos de carbono, 43-47 \% siendo los valores recomendados $50-55 \%$, un exceso de alimentos ricos en proteínas, $25 \%$ siendo los valores recomendados 10-15 $\%$, y unos porcentajes normales de grasas totales (30-35\%), aunque con porcentajes de ácidos grasos saturados demasiados elevados (tabla 3) por la ingesta de comidas rápidas. Este tipo de comidas podría indicar una socialización por imitación entre sus iguales. En general, predominan las comidas en casa con la familia o sólo (1:1), hecho relevante que se compagina comidas fuera de casa con amigos (tabla 5) y que justificó el menor consumo de patata e inmediatamente el menor consumo de los alimentos ricos en hidratos de carbono en la dieta. Porque el adolescente no cocina hervidos o platos de olla para comer sólo o con amigos.

Referente a los hábitos de alimentación de los adolescentes, en el entorno rural cabe destacar el aumento de comidas en casa con la familia, en ocasiones con los amigos y limitando las salidas a comer (tabla 5). Las comidas rápidas se suele apreciar en el individuo sólo o acompañado por amigos, en casa propia o en casa de estos. El consumo de hidratos de carbono es proporcionado por una mayor ingesta de patata y dulces aunque en el caso de los dulces son hidratos de carbono simples que son peligrosos en exceso (las recomendaciones son un máximo de un $10 \%$ del $50-55 \%$ de la totalidad de la ingesta de hidratos de carbono); el consumo de grasas está aumentado por la mayor ingesta de mantequillas y frutos secos, el exceso de grasas saturadas esta conseguido por la mantequilla; y como bebidas los zumos son seleccionados con más asiduidad.

En los hábitos de alimentación del entorno urbano, se observa como predominan menos las costumbres propias de la zona porque las comidas en casa se hacen con la familia o sólo (1:1), a parte de que el efecto de socialización es mayor por las 
comidas con amigos (tabla 5). El porcentaje de alimentos ricos en proteínas es mayor en parte por el consumo mayor de jamón serrano, $25 \%$ cuando la normalidad se establece entorno a 10-15\%, el porcentaje de hidratos de carbono y grasas se verá aumentado con la ingesta de bollería. La ingesta de colesterol es elevada, el mayor consumo de jamón serrano y grasas aumentará este valor. Y por último, se supone que las costumbres menos arraigadas se le sumaría una mayor edad, dieciocho años exactamente, que podría valorarse como consecuencia de una disminución de comidas de olla por menor uso de patatas y una mayor utilización de comidas rápidas.

Referente a la alimentación de los niños y los adolescentes, los niños manifiestan un mejor cumplimiento de las recomendaciones de macronutrientes (hidratos de carbono, grasas y proteínas) (tabla 3), siendo los padres responsables de esta selección de alimentos, aunque su desayuno y merienda-cena no son adecuados hacen mayor número de comidas en su casa. Los adolescentes reflejarían una mayor calidad en la alimentación en cuanto al números de comidas si no fuera por las que en casa de amigos o fuera tipo, todas ellas tipo comidas rápidas.

Se destaca una trasgresión de los hábitos de alimentación de los adolescentes porque realizan una mejor calidad en las comidas (tabla 4), alterando este equilibrio los fines de semana. Dónde se come parece que está menos claro que en los niños de 12 años (tabla 5).

En general, se plantea un déficit de verduras, de frutas, pan y pescados puesto que se consumió aproximadamente _ parte de lo que se consume de carnes; y excesos de productos de bollería de chocolate, de zumos y carnes (tabla 2). Respecto a la valoración general de la calidad de las comidas, destacar que en la población urbana (comparación entre niños y adolescentes) los hábitos de alimentación son más heterogéneos que en la población rural. Por otro lado, se da mayor homogeneidad entre los adolescentes como comparación de los niños por un lado y los adolescentes por otro. Respecto a donde y con quién comen, las diferencias generales existen con "donde comen", ya que los adolescentes reflejan mayores diferencias de alimentación que los niños y la misma situación en el entorno rural con respecto al entorno urbano.

\section{DISCUSIÓN Y CONCLUSIONES}

En primer lugar, en relación con el método utilizado para registrar los hábitos de alimentación, la cumplimentación de los carnets alimentarios directamente por los encuestados, se ha mostrado eficaz, aunque puede tener errores en cuando a la cantidad de alimentos, no así en cuanto a las veces que los ingieren, válido por tanto para estudios semicuantitativos y cualitativos de los hábitos alimentarios (plenufar). También cabe destacar el error de cuantificación de aceite o grasas añadidas en las comidas, puesto que es un ingrediente no visible, que es únicamente valorable por la que cocine y que puede esta relacionado directamente con los resultados de déficit de energía en la dieta.

El número de comidas indica que en todos los casos del entorno rural prácticamente se realizaron las tradicionales comidas como: desayuno, almuerzo, merienda y cena (alrededor de 27 comidas semanales), a diferencia de la población urbana (alrededor de 25-26 comidas semanales) en la que se merienda-cena y por lo tanto la pérdida de una comida, la cena (tabla 4).

Referente a la comparación de los niños y los adolescentes en cuanto a la norma de frutas y verduras, son porcentajes encontrados en el análisis de datos de Krebs-Smith y cols se refiere a 3.140 niños y adolescentes de edades entre 2 y 18 años, en los que el aporte de frutas y de vegetales era muy bajo en relación con las recomendaciones. Un $56 \%$ de los varones y un $63 \%$ de las muchachas entre 12 y 18 años tomaban menos de una porción de fruta por día y en cuanto a los vegetales los porcentajes de menos de una vez por día eran del 8.6 y $13.2 \%$, respectivamente. El bajo consumo de frutas y verduras, como fuente esencial de fibras y vitaminas, $\mathrm{y}$ factor diferencial de forma primordial de la "dieta mediterránea" y de sus ventajas (Fiol C.1996, Fischler.1996, Maldonado P.1991). En la evolución del consumo de la alimentación del ministerio de agricultura, pesca y alimentación destaca una disminución de la ingesta de los hidratos de carbono, entorno a un 8-13\% en niños y 11 $15 \%$ en adolescentes; y un aumento en las proteínas, $12-13 \%$ en niños y $10-11 \%$ en adolescentes según la recomendaciones de la O.M.S. El aumen- 
to de la ingesta de hidratos de carbono de cadena larga significaría las veces que los niños y adolescentes toman una comida de "cuchara" o "comida caliente". (Barrabriga A 1998).

Referente a la comparación de los adolescentes, las muchachas adolescentes sólo consumían diariamente un $80 \%$ de la cantidad de energía recomendable para su edad (Barrabriga A 1998).

Las modificaciones de los hábitos alimentarios fueron comunes a las dos poblaciones, rural y urbana, igualándose éstos entre ambas poblaciones como efecto de la uniformidad cultural, efectos extensibles a los niños y adolescentes.

En los niños fue latente la búsqueda de rapidez y comodidad en su alimentación para los padres con los desayunos rápidos y las meriendas-cenas. Característica potenciada por el entorno urbano, al igual que la descompensación de los macronutrientes. Se ha encontrado en ambos entornos una similitud de dónde y con quién comen.

En los adolescentes se destaca una mayor calidad en las comidas entre semana, cuestión que es modificada los fines de semana por la ingesta de comidas rápidas en casa de amigos.

Se muestra un consumo bajo de pescado, frutas y verduras, lo que puede presentar problemas de salud a medio y largo plazo, factor diferencial de forma primordial de la "dieta mediterránea" y de sus ventajas (Fiol C. 1996, Fischler. 1996, Maldonado P. 1991). La disminución de la ingesta de alimentos ricos en hidratos de carbono de cadena larga y el aumento de alimentos ricos en proteínas es una realidad hoy en día (Moreiras O. 1989, Rizo M. 2001).

Y por último, a pesar de que los carnets alimentarios de una semana puede tener de valoración de las cantidades de los alimentos como las ingesta de grasas añadidas, pueden servir para detectar desviaciones poblacionales con una mayor exactitud que los estudios de 24 horas (Hetzler AA. 1993, Jhonson RK. 1991).

\section{BIBLIOGRAFÍA}

- ALARCÓN N, ACOSTA JA (2001). Trabajo fin de carrera: Trastornos de la alimentación: bulimia nerviosa. Universidad Católica San Antonio de Murcia. Murcia.

- ALONSO V, MORALES MM Y CANDENAS MA (1991). Comparación de dos tipos de encuestas dietéticas. Enfermería Científica 132: 17-24.
- BALlABRIGA A, CARRASCOSA A (1998). Nutrición en la infancia y adolescencia. Ergon. Madrid.

- BREWSTER L AND JACOBSON HF (1978). The changing american diet. Washington Center form Science in the Public Interest.

- CASTELLS M (1995). La ciudad informacional: tecnología de la información, reestructuración económico y el proceso urbano regional. Editorial Alianza Editorial. Madrid.

- CONTRERAS J (1993). Antropología de la alimentación. Ediciones de la Universidad Complutense. Madrid.

- Consejo general de colegios oficiales de farmacéuticos. Educación del farmacéutico: Plenufar 2. Edufar.

- FIOL C (1996). Los alimentos en la dieta mediterránea. En: "La alimentación mediterránea: Historia, cultura, nutrición". Institut Cátala de la Mediterránia. Barcelona. Icaria.

- FISCHLER (1996). El modelo alimentario mediterráneo: mito y/o realidad. En: "La alimentación mediterránea". Barcelona. Icaria.

- GRACIA M. (1996) Paradojas de la alimentación contemporánea. Icaria. Institut Cáala d’Antropología. Barcelona.

- GRACIA M (1996) Antropología de la alimentación. En: Ensayos de Antropología Cultural. J Prat y A Martinéz Eds. Editorial Ariel. Barcelona.

- HETZLER AA, BOWENS J, HULL S (1993). Preschoolers reporting of food habits. J Am Diet Assoc; 93: 1159-61.

- JHONSON RK, DRISCOL P, GORAN MI (1996): Comparison of multiple-pass 24-hour-recall estimates of energy intake total energy expenditure determined by the doubly labeled water method in young children. J Am Diet Assoc; 96: 1140-4.

- MALDONADO P, TÓRTOLA V, MARTÍNEZ J, et al (1991). Proyecto de intervención comunitaria a partir de un análisis de dietas escolares. Enfermería Científica 172-173: 39-41.

- MARTÍ-HENNEBERG C, CAPDEVILA F. Tratado de Nutrición Pediátrica. Ediciones Doyma S.L. Barcelona. 5: 57-64. Ministerio de Agricultura, Pesca y Alimentación (1996). La alimentación en España 1995.

- MARTI-HENNEBERG C, SALAS J (1987). Evolución del consumo nutricional en España durantes los últimos 25 años. Med Clin 88: 369-371. Barcelona

- MATAIX J, MARTINEZ E, MAÑAS MARIANO (Versión 0698.046). Programa de Alimentación y Salud. Instituto de Nutrición y tecnologías de alimentos. Universidad de Granada.

- MEDINA FX (1996). Alimentación, dieta y comportamientos alimentarios en el contexto mediterráneo. En: "La alimentación mediterránea". Barcelona. Icaria.

- MOREIRAS O, VARELA G (1989). The mediterranean diet in Spain. Eur J Clin Nutr. 43: 83-87. 
- MOREIRAS O, CARBAJAR A, PEREA I. Evolución de los hábitos alimentarios en España (1990). Ministerio de Sanidad y Consumo. Madrid.

- RIZO M, CORTES E, HERNÁNDEZ C, MOYA M (1990). "Análisis preliminar para la puesta en marcha de un programa de salud de nutrición infantil". Atención primaria, 7, 4; 278-282.

- RIZO M, CORTES E (1990). "Resultados obtenidos por enfermería pediátrica en un programa de atención primaria en nutrición”. Enfermería científica 96: 32-35.

- RIZO M, CORTÉS E, RUÍZ MT (1988). "Diferencias entre la dieta infantil según el estado nutricional en la población urbana". Enfermería científica, 190-191; 47-51.

- RIZO M, INFANTES MJ, JUSTAMANTE (2001). "Uniformidad de la cultura mediterránea entre la población urbana y rural”. Revista 50. Vol. 5. Pág. 6-11. Noviembre 2002.

- RIZO M, INFANTES MJ, JUSTAMENTE J (2001). "Dieta mediterránea en poblaciones urbanas y rurales". IV Congreso Nacional de Enfermería de la infancia, XIII Jornadas Nacionales de Enfermería de la infancia. Sevilla 2001. (globalización comentado en la introducción)

- RODRÍGUEZ P (2001). Tendencia del consumo de alimentos en Murcia y relación con la dieta mediterránea. Editado por A.G. Novograf. (Consejería de Sanidad y Consumo. Dirección general de Salud Pública). Murcia.
- RODRÍGUEZ F, BANEGAS JR, GRACIANI MA, HERNÁNDEZ R, REY CALERO J (1996). El consumo de alimentos y nutrientes en España en el período 19401988. Análisis de su consistencia con la dieta mediterránea. Med Clin 106: 161-168. Barcelona.

- RODRÍGUEZ ARTALEJO F, GRACIANI MA, BANEGAS JR, MARTÍN-MORENO JM, SABATÉ J, RESY CALERO J (1996). El consumo de alimentos y nutrientes en España en el período 1940-1988 (y II). Un estudio comparativo de las principales fuentes de información sobre el consumo alimentario. Med Clin 107: 446-452. Barcelona.

- SABATÉ F (1984). Las encuestas de alimentación y nutrición en España. Bibliografía 1939-1970. San Hig Pub 58: 1.117-1.132.

- SERRA L, RIBAS L, LLOVERAS G, SALLERAS L (1993). Changing patterns of fat consumption in Spain. Eur J Clin Nutr 47 (Supl 1): 13-20.

- TOJO R, MATAIX J, ROS L, TORMO R, MOYA M, GIOVANNINI M, LEIS R, POMBO M, VICTORIA J.C (2001). Tratado de Nutrición Pediátrica. Ediciones Doyma S.L. Barcelona.

- VILLABÍ JR, MALDONADO R (1988). La alimentación en España desde la postguerra hasta los años ochenta. Revisión de encuestas. Med Clin. Barcelona. 90: 127-130.

- WILLET MC, SAMPSON L, STAMPSON L, SATAMPFER MJ, et al (1985). Reproductibility and validity of a semiquantitative food frequency questionari. Am J Epidemiol 124: 17-27.

Tabla N ${ }^{\circ}$ 1: Descripción de dos grupos: en edades y entornos

\begin{tabular}{|c|c|c|c|c|c|c|}
\hline & \multicolumn{3}{|c|}{ Rural } & \multicolumn{3}{|c|}{ Urbano } \\
\hline & Niños $(\mathrm{n}=25)$ & $\mathrm{p}$ & Adolescentes $(\mathrm{n}=26)$ & Niños $(\mathrm{n}=25)$ & $\mathrm{p}$ & Adolescentes $(\mathrm{n}=28)$ \\
\hline & Media (SD) & & Media (SD) & Media (SD) & & Media (SD) \\
\hline Edad & $12.1 \quad(0.4)$ & 0 & $17.9 \quad(0.9)$ & $12.0(0.2)$ & 0 & $18 \quad(0)$ \\
\hline Sexo & $1.6 \quad(0.5)$ & 0 & $\begin{array}{ll}1.8 & (0.4)\end{array}$ & $1.8(0.4)$ & $\mathrm{ns}$ & $\begin{array}{ll}1.8 & (0.4)\end{array}$ \\
\hline Talla & $153.4 \quad(7.5)$ & $\mathrm{ns}$ & $170.7(7.7)$ & $149.0(8)$ & $\mathrm{ns}$ & $169.2(7.2)$ \\
\hline Peso & $47.2 \quad(9.4)$ & $\mathrm{ns}$ & $61.0 \quad(9.9)$ & $42.0(7.2)$ & $\mathrm{ns}$ & $\begin{array}{ll}59.6 & (9.9)\end{array}$ \\
\hline IMC & $20.1 \quad(3.8)$ & $\mathrm{ns}$ & $21.0 \quad(3.2)$ & $18.8(2.6)$ & $\mathrm{ns}$ & $20.7 \quad(2.5)$ \\
\hline
\end{tabular}

* ns. : no significativo 
Tabla $\mathrm{N}^{\circ}$ 2: Descripción de dos entornos: Comidas y distintos alimentos ingeridos

\begin{tabular}{|c|c|c|c|c|c|c|}
\hline & \multicolumn{3}{|c|}{ Rural } & \multicolumn{3}{|c|}{ Urbano } \\
\hline & Niños & \multirow[t]{2}{*}{$\mathrm{p}$} & Adolescentes & Niños & \multirow[t]{2}{*}{$\mathrm{p}$} & Adolescentes \\
\hline & Media (SD) & & Media (SD) & Media (SD) & & Media (SD) \\
\hline Comidas & $27.1(2.7)$ & 0 & $27.2(4)$ & $26.2(4.6)$ & ns & $25.4(4.3)$ \\
\hline Lácteos/Deriv. & $8.7 \quad(3.5)$ & ns & $8.6 \quad(4.39)$ & $11.0(3.9)$ & ns & $10.0(3.2)$ \\
\hline Verduras & $5.9(5.0)$ & ns & $7.8 \quad(5.4)$ & $2.92(4.3)$ & ns & $7.4 \quad(5.1)$ \\
\hline Frutas & $4.0 \quad(4.0)$ & ns & $1.3 \quad(2.2)$ & $2.6(2.9)$ & ns & $2.5 \quad(3)$ \\
\hline $\mathrm{Pan} / \mathrm{Cer} / \mathrm{Ar} / \mathrm{P} /$ & $11.0(4.0)$ & ns & $12.0(5.0)$ & $13.0(5)$ & ns & $14.0(5)$ \\
\hline Car/Pesc/Leg/huev/ & $11.0(3.0)$ & ns & $9.0 \quad(3)$ & $7.0(3)$ & ns & $12.0(3)$ \\
\hline Gras/Ac/Dulces & $7.0(5.0)$ & ns & $9.0 \quad(6)$ & $6.0(4)$ & ns & $6.0 \quad(5)$ \\
\hline
\end{tabular}

\section{Tabla $\mathrm{N}^{\circ}$ 3: Macronutrientes de los distintos entornos y edades}

\begin{tabular}{|l|l|l|l|l|}
\hline \multirow{2}{*}{$\%$ Macronutrientes } & \multicolumn{2}{|c|}{ RURAL } & \multicolumn{2}{c|}{ URBANO } \\
\cline { 2 - 5 } & niños & adolescentes & niños & adolescentes \\
\hline$\%$ H.C.: & $50 \%$ & $47 \%$ & $45 \%$ & $43 \%$ \\
\hline$\%$ Proteínas: & $21 \%$ & $22 \%$ & $24 \%$ & $25 \%$ \\
\hline$\%$ Grasas: & $29 \%$ & $31 \%$ & $30 \%$ & $32 \%$ \\
\hline$\%$ Grasas Monoinsat.: & $50 \%$ & $46 \%$ & $44 \%$ & $44 \%$ \\
\hline$\%$ Grasas Poliinsat.: & $21 \%$ & $14 \%$ & $14 \%$ & $15 \%$ \\
\hline$\%$ Grasas Saturada: & $29 \%$ & $40 \%$ & $41 \%$ & $41 \%$ \\
\hline Colesterol & $304 \mathrm{mg}$ & $289 \mathrm{mg}$ & $248 \mathrm{mg}$ & $327 \mathrm{mg}$ \\
\hline
\end{tabular}


Tabla No ${ }^{\circ}$ : Calidad de Comidas de los distintos entornos y edades

\begin{tabular}{|c|c|c|c|c|c|c|}
\hline \multirow{2}{*}{$\begin{array}{l}\text { Rural/ } \\
\text { Urbano }\end{array}$} & \multicolumn{3}{|c|}{ Comidas: Niños } & \multicolumn{3}{|c|}{ Comidas: Adolescentes } \\
\hline & Media & SD & $\mathrm{p}$ & Media & SD & $\mathrm{p}$ \\
\hline P.fritras & 0.7 & 0.8 & ns. & 0.9 & 1.0 & ns \\
\hline Papas & $\begin{array}{l}0.0 \\
0.1\end{array}$ & $\begin{array}{l}0.0 \\
0.3\end{array}$ & 0.00 & $\begin{array}{l}1.1 \\
0.2\end{array}$ & $\begin{array}{l}1.7 \\
0.8\end{array}$ & 0.07 \\
\hline D.óptimo & $\begin{array}{l}0.5 \\
1.5\end{array}$ & $\begin{array}{l}0.7 \\
2.0\end{array}$ & 0.00 & $\begin{array}{l}1.4 \\
1.6\end{array}$ & $\begin{array}{l}2.1 \\
2.0\end{array}$ & ns \\
\hline Desayuno & $\begin{array}{l}6.0 \\
5.2\end{array}$ & $\begin{array}{l}1.4 \\
2.0\end{array}$ & $\mathrm{~ns}$ & $\begin{array}{l}4.4 \\
4.4\end{array}$ & $\begin{array}{l}2.7 \\
2.2\end{array}$ & $\mathrm{~ns}$ \\
\hline Almuerzo & $\begin{array}{l}1.6 \\
1.0\end{array}$ & $\begin{array}{l}2.0 \\
1.5\end{array}$ & 0.05 & $\begin{array}{l}1.2 \\
1.5\end{array}$ & $\begin{array}{l}1.7 \\
1.5\end{array}$ & ns \\
\hline A.Rápido & $\begin{array}{l}0.5 \\
0.3\end{array}$ & $\begin{array}{l}1.0 \\
0.8\end{array}$ & ns & $\begin{array}{l}0.0 \\
1.8\end{array}$ & $\begin{array}{l}0.0 \\
8.0\end{array}$ & 0.02 \\
\hline Merienda & $\begin{array}{l}1.6 \\
5.2\end{array}$ & $\begin{array}{l}2.4 \\
2.2\end{array}$ & ns & $\begin{array}{l}2.9 \\
2.7\end{array}$ & $\begin{array}{l}2.4 \\
2.3\end{array}$ & $\mathrm{~ns}$ \\
\hline Mer.cena & $\begin{array}{l}4.0 \\
0.3\end{array}$ & $\begin{array}{l}2.8 \\
0.7\end{array}$ & 0.00 & $\begin{array}{l}0.4 \\
0.7\end{array}$ & $\begin{array}{l}0.6 \\
1.6\end{array}$ & 0.01 \\
\hline Cena & $\begin{array}{l}2.1 \\
6.5\end{array}$ & $\begin{array}{l}2.9 \\
0.8\end{array}$ & 0.00 & $\begin{array}{l}6.0 \\
6.0\end{array}$ & $\begin{array}{l}0.9 \\
2.0\end{array}$ & 0.01 \\
\hline Cena.col & $\begin{array}{l}6.5 \\
3.0\end{array}$ & $\begin{array}{l}0.8 \\
3.1\end{array}$ & 0.00 & \begin{tabular}{|l|}
0.1 \\
0.4
\end{tabular} & $\begin{array}{l}0.3 \\
0.8\end{array}$ & 0.00 \\
\hline
\end{tabular}


Tabla $\mathrm{N}^{\circ} 5$ : Con quién y Donde comen de los distintos entornos y edades

\begin{tabular}{|c|c|c|c|c|c|c|}
\hline \multirow{2}{*}{$\begin{array}{l}\text { Con Quién } \\
\text { Dónde }\end{array}$} & \multicolumn{3}{|c|}{ Comidas: Niños } & \multicolumn{3}{|c|}{ Comidas: Adolescentes } \\
\hline & Media & SD & $\mathrm{p}$ & Media & SD & $\mathrm{p}$ \\
\hline Familia & $\begin{array}{l}5.8 \\
12.9\end{array}$ & $\begin{array}{l}6.6 \\
7.8\end{array}$ & ns & $\begin{array}{l}6.7 \\
9.3\end{array}$ & $\begin{array}{l}4.68 \\
5.527\end{array}$ & ns \\
\hline Padres & $\begin{array}{l}3.3 \\
1.6\end{array}$ & $\begin{array}{l}4.5 \\
2.9\end{array}$ & 0.04 & $\begin{array}{l}2.3 \\
0.9\end{array}$ & $\begin{array}{l}3.672 \\
1.69\end{array}$ & 0.0 \\
\hline Madres & $\begin{array}{l}4.9 \\
1.9\end{array}$ & $\begin{array}{l}6.3 \\
3.2\end{array}$ & 0.01 & $\begin{array}{l}1.3 \\
2.0\end{array}$ & $\begin{array}{l}2.637 \\
2.799\end{array}$ & ns \\
\hline Hermanos & $\begin{array}{l}4.6 \\
2.1\end{array}$ & $\begin{array}{l}10.4 \\
2.4\end{array}$ & ns & $\begin{array}{l}0.6 \\
0.7\end{array}$ & $\begin{array}{l}1.372 \\
1.069\end{array}$ & 0.05 \\
\hline Abuela & $\begin{array}{l}0.4 \\
0.3\end{array}$ & $\begin{array}{l}1.2 \\
1.0\end{array}$ & ns & $\begin{array}{l}0.3 \\
0.0\end{array}$ & $\begin{array}{l}1.013 \\
0\end{array}$ & ns \\
\hline Familiares & $\begin{array}{l}0.4 \\
0.8\end{array}$ & $\begin{array}{l}0.9 \\
1.4\end{array}$ & ns & $\begin{array}{l}0.0 \\
0.1\end{array}$ & $\begin{array}{l}0.189 \\
0.400\end{array}$ & ns \\
\hline Amigos & $\begin{array}{l}2.2 \\
0.6\end{array}$ & $\begin{array}{l}2.4 \\
1.0\end{array}$ & 0.00 & $\begin{array}{l}4.4 \\
2.7\end{array}$ & $\begin{array}{l}2.818 \\
2.116\end{array}$ & ns \\
\hline Solo & $\begin{array}{l}4.6 \\
3.5\end{array}$ & $\begin{array}{l}4.2 \\
4.4\end{array}$ & ns & $\begin{array}{l}6.5 \\
5.8\end{array}$ & $\begin{array}{l}2.715 \\
3.912\end{array}$ & 0.03 \\
\hline Casa & $\begin{array}{l}21.4 \\
21.7\end{array}$ & $\begin{array}{l}4.0 \\
4.6\end{array}$ & ns & $\begin{array}{l}17.7 \\
19.0\end{array}$ & $\begin{array}{l}2.667 \\
5.492\end{array}$ & 0.00 \\
\hline Casa abuela & $\begin{array}{l}0.8 \\
0.9\end{array}$ & $\begin{array}{l}1.3 \\
1.4\end{array}$ & ns & $\begin{array}{l}0.4 \\
1.2\end{array}$ & $\begin{array}{l}1.367 \\
4.628\end{array}$ & ns \\
\hline Casa fuera & $\begin{array}{l}0.2 \\
0.5\end{array}$ & $\begin{array}{l}0.5 \\
0.9\end{array}$ & 0.00 & $\begin{array}{l}0.0 \\
0.0\end{array}$ & $\begin{array}{l}0 \\
0.2\end{array}$ & 0.03 \\
\hline Casa amigo & $\begin{array}{l}0.2 \\
0.2\end{array}$ & $\begin{array}{l}0.4 \\
0.5\end{array}$ & ns & $\begin{array}{l}0.2 \\
0.5\end{array}$ & $\begin{array}{l}0.68 \\
1.005\end{array}$ & 0.03 \\
\hline Fuera F & $\begin{array}{l}0.4 \\
0.6\end{array}$ & $\begin{array}{l}1.4 \\
2.2\end{array}$ & ns & $\begin{array}{l}0.5 \\
0.2\end{array}$ & $\begin{array}{l}1.26 \\
0.374\end{array}$ & 0.01 \\
\hline Fast. F & $\begin{array}{l}0.4 \\
0.1\end{array}$ & $\begin{array}{l}0.7 \\
0.4\end{array}$ & ns & $\begin{array}{l}0.3 \\
0.2\end{array}$ & $\begin{array}{l}0.659 \\
0.436\end{array}$ & ns \\
\hline Colegio & $\begin{array}{l}1.4 \\
0.3\end{array}$ & $\begin{array}{l}2.2 \\
1.0\end{array}$ & 0.00 & $\begin{array}{l}3.0 \\
1.3\end{array}$ & $\begin{array}{l}2.457 \\
1.547\end{array}$ & 0.03 \\
\hline Parque & $\begin{array}{l}0.8 \\
0.7\end{array}$ & $\begin{array}{l}1.9 \\
1.3\end{array}$ & ns & $\begin{array}{l}1.0 \\
0.9\end{array}$ & $\begin{array}{l}1.503 \\
1.424\end{array}$ & ns \\
\hline
\end{tabular}

ns: no significativo 